\title{
Improving Medication Reconciliation on the Surgical Wards of a District General Hospital
}

Erika Hughes, Paul Hegarty, Andrew Mahon

\begin{abstract}
During a routine audit it was noted that the surgical wards were consistently underperforming in their rate of medicines reconciliation in comparison to other specialities. The process of medication reconciliation is usually performed by junior doctors during the admission process and can be a complex task which is usually undertaken in the midst of several other jobs. The aim of this project was to review this process and identify methods of improving patient safety. This led to the design of a surgical admissions proforma which incorporated a 'medications on admission' section, to be used for reconciliation. Over a six month period from its introduction into a pilot ward it was noted to improve medication reconciliation from $60 \%$ to $85 \%$. The benefits were discussed with members of the trust and a standardised version of the admissions proforma has since been rolled out to all hospitals within the trust.
\end{abstract}

\section{Problem}

An on-going audit on medication reconciliation, comparing the medical, surgical and trauma and orthopaedics wards in a district general hospital in Belfast, Northern Ireland, highlighted consistently lower medication reconciliation statistics in surgery compared with the rest of the hospital.

Although there were no reports of critical incidents occurring this could still have serious implications for patients, with an increased risk of preventable adverse drug events (pADE), for example, the accidental omission of a drug during the admission process, or failing to restart at discharge an essential drug which was temporarily stopped prior to surgery. The risk of this increases further with the high patient turnover in surgical wards and polypharmacy in elderly populations.

\section{Background}

Medication errors are the leading cause of injury to hospital patients, resulting in increased morbidity and mortality and prolongation of hospital stay. (1) Half of all errors occur at the interface of care, with the majority being omission of a drug. (2) Guidance outlining ways to reduce the risk of medication error at care interfaces was published in 2006 by the Royal Pharmaceutical Society of Great Britain and since then multiple organisations, including the National Institute for Health and Clinical Excellence and the National Patient Safety Agency, have published documents aimed at highlighting the need for improvement in medication reconciliation. $(3,4,5)$

Medication reconciliation is the process whereby the most accurate list of a patient's medications is created, including drug name, dosage, frequency and administration route. This is then compared to the admission, transfer or discharge documentation, discrepancies are identified and changes documented, resulting in a complete and accurate list. (6)

\section{Baseline Measurement}

From July 2010 a monthly audit was performed assessing medication reconciliation in medicine, surgery and trauma \& orthopaedics in the Ulster Hospital. Twenty patients' notes were audited per month in each specialty. Notes were picked at random across the wards and checked to see if medications had been reconciled within 24 hours of admission.

Surgery consistently performed below the rest of the hospital with an average medication reconciliation of $60 \%$ compared with approximately $80 \%$ for the rest of the hospital.

See supplementary file: Baseline Measurements.pdf

\section{Design}

Following the new design of the MOA form, a trial was carried out amongst junior doctors to test how it compared with the previous version. Feedback received was positive for the most part, with it described as easier to use and understand. However many users stated that the main problem was not the form, but convincing junior staff to seek out and complete extra paperwork on top of an already busy schedule.

\section{Strategy}

Using the feedback from our initial cycle the team decided to create a surgical admissions proforma. This would combine all documentation needed for admission as was already the case in the medical wards. Over the next month this was developed and a pilot commenced on one of the elective surgical wards. The process measures identified the majority of admissions were performed by junior staff; this information was used to target education at this 
population to help improve compliance.

\section{Post-Measurement}

Results were gathered on a monthly basis, allowing the intervention and performance of further PDSA cycles as necessary to improve the chance of reaching the previously outlined objective. The main outcome measure was percentage patients with all medicines reconciled within 24 hours of admission. Further to this there were process measures that would help target any further interventions that might be needed. These included; who was the admitting clinician, was the MOA form filled in, was allergy status completed and was the source of medication history indicated?

Twenty charts were audited per month in the pilot ward, five per week, chosen at random. This was then compared with the rest of the surgical wards. In the first month after implementation of the proforma, medication reconciliation increased from $60 \%$ to $80 \%$. This trend continued, with the pilot ward consistently outperforming the rest of the surgical wards.

However, after the six months the pilot ward failed to meet the target of $95 \%$ medication reconciliation, instead achieving a maximum of $85 \%$. A number of contributing factors led to this, including the fact that not all admissions on the pilot ward used the proforma, but were still included in data collection due to randomisation. When looking specifically at data for when the proforma was used it was noted to have $100 \%$ medication reconciliation over two consecutive months.

\section{Lessons and Limitations}

1. Having a multidisciplinary team involved in the project is key to success. There are many barriers to overcome but involvement of the whole team can help to limit these, such as nursing staff on the wards to ensure proformas are available for use, doctors to gain permission for pilot from senior staff and to educate colleagues and pharmacy to help in the design and assessment of interventions. 2. Difficulties were noted around the time of changeover for junior doctors; a dip can be noticed in the statistics. This could have been overcome with a short induction session to educate the staff in the use and importance of the proforma.

3. Having varied admission practice over the numerous surgical wards made it increasingly difficult to ensure proper completion of the admissions proforma on the pilot ward.

\section{Conclusion}

Medication reconciliation was noted to be considerably lower in surgery for over a year with little improvement of any interventions thus far, with a medication on admissions form that many did not understand and most did not use. This was putting many patients at risk of preventable adverse drug events.

What started off as an analysis into the cause of underperformance slowly became a myriad of solutions which were implemented over a number of months; the skill mix in the team allowed effective critique of what would and wouldn't work, and as mentioned in previous projects, the engagement of a senior management figure has provided a link to allow change to become implemented successfully.

The initial target of $95 \%$ medication reconciliation was not achieved in the assigned 6 month timeframe. Nevertheless, significant improvements could be noted with the introduction of the admissions proforma. Looking at the results, the rate of medicines reconciliation on the pilot ward was $100 \%$, when the proforma was utilised, for two consecutive months. However, when including nonproforma admissions this rate fell to $85 \%$. This was interpreted as a demonstration of the effectiveness of the documentation and further confirms the need for standardisation across all specialties to reduce prescribing errors. It also indicates the need for further encouragement to be given to admitting physicians to utilise the documentation.

Following this audit, the trust has implemented standardised admission documentation in all specialties throughout its hospitals. Despite the success of the project, work must continue to educate medical staff, who rotate between all trusts throughout Northern Ireland on a regular basis, to ensure the continuity of high standards achieved thus far. One possible solution for this would be regional standardisation of admission documentation which can hopefully be achieved in the near future.

\section{References}

1. Pirmohamed, M., James, S., Meakin, S., Green, C., Scott, A. K., Walley, T. J., Farrar, K., Park, B. K. \& Breckenridge, A. M. (2004) Adverse drug reactions as cause of admission to hospital: prospective analysis of 18820 patients. British Medical Journal, 329, 15-19.

2. Rozich, J. D. \& Resar, R. K. (2001) Medication safety: one organization's approach to the challenge. Journal of Clinical Outcomes Management, 8 (10), 27-34.

3. The Royal Pharmaceutical Society of Great Britain. Moving patients medicines safely: Guidance on Discharge and Transfer Planning. London: RPSGB; 2006.

4. National Institute for Health and Clinical Excellence. Technical Patient Safety Solutions For Medicines Reconciliation On Admission of Adults to Hosptial. London: NICE; 2007.

5. National Patient Safety Agency [Internet]. London: Patient Safety - Medication Safety [updated 2012 Mar 22; cited 2012 December 1]. Available from: http://www.nrls.npsa.nhs.uk/resources/patient-safetytopics/medication-safety/

6. Manno, M. S. \& Hayes, D. D. (2006) How medication reconciliation saves lives. Nursing, 36 (3), 63-64.

7. Coombes I, Stowasser D, Reid C, Mitchell C (2009) Impact of a standard medication chart on prescribing errors: a before and after audit. Qual Safety Healthcare 18: 478-485 